\title{
Optical part measuring inside a milling machine
}

\author{
Nicolas Meier, M.Sc. ${ }^{1}$; Prof. Dr. Anthimos Georgiadis ${ }^{1}$ \\ ${ }^{1}$ Leuphana University of Lueneburg, Institute of Product and Process Innovation (PPI), Volgershall 1 , \\ 21339 Lueneburg, Germany \\ Corresponding author: nicolas.meier@leuphana.de
}

\begin{abstract}
The aim of the work is to measure the dimensions of a workpiece inside a milling machine with an optical system. The process of milling should be interrupted for the measuring process but without reclamping. After that, the measurement system should give a feedback to the controller of the milling machine, so that the process could be readjusted on the basis of the measurement results. With this process, errors should be detected very early and the quality of the workpiece should be improved.

The aim is to measure 3 dimensions of the workpiece (height, width and length) with a sensor system. A laser triangulation system has been used for the experiments. The sensor scans the object only in two dimensions. Because of that, a further relative movement is necessary. For the experiment, the movement was realized by the forward movement of the table of the milling machine. Current measurements show that the sensor system is able to work inside the production area and while the milling machine is running.

Furthermore, it comes out, that the resolution of the height is in the range of a digit micrometer or in a lower double-digit micrometer range. The resolution of the length lies in the same range like those of the height measurements. The resolution of the width is much less, about 20 micrometers. In order to get higher resolution, a second sensor, which scans the workpiece from the side, is going to be used.
\end{abstract}

Key words: Zero defect manufacturing, optical laser inspection system.

\section{Motivation and reasons for the research in this field}

In the context of the innovation campaign "Produktion 2020" it is a common goal of research and industry to increase the added value in the field of production up to $30 \%$ [1]. This results in two important areas for industry and research. Firstly, the integration of measurement technology into the production environment for the generation of valid measurements about the production process should be increased. On the other hand, existing data should be increasingly used by the automated processing and recycling into the production process [2].

The work which was done previously is concerned with the first goal. The aim is to integrate an optical sensor system into a milling machine. The work that will follow in the future is concerned with the second goal. The aim is to connect the data of the measurement system with the controller of the milling machine.
Depending on the implementation, the integration of the process can be distinguished between offline, in-situ and in-process strategies [3].

In the performed test a sensor was integrated into the production machine. Machining and measuring run separately from each other. In contrast to the off-line monitoring, the workpiece is not removed from the machine. Based on the measurement results it is possible to rework the workpiece in a further processing step. A costly recalibration of the workpiece into the machine coordinate system is not necessary. In addition, the time of the feedback can be reduced significantly [4].

Because of the listed characteristics it becomes clear, that the performed measurement is an insitu strategy. For the in-situ solution it is possible to use scanning sensors. This can be achieved by the temporal separation between measurement and production. In addition there is the possibility to realize a spatial separation between the sensor and the tool inside of the milling machine. This allows a better protection 
for the sensor against contamination and damage.

\section{Description of the used sensor}

The sensor system which was used is a $3 D$ laser inspection system. It is a system which works with a blue laser. A sharp line beam is formed on the light-receiving element by focusing a short wavelength $405 \mathrm{~nm}$ laser to its diffraction limit with a 2D lens. This generates a stabilized high-precision profile ensuring optimal accuracy and limited image blur. The output intensity of the laser source provides a clear return even on targets with a wide range of reflectivity.

The measurement principle of the sensor works according to the principle of laser triangulation:

A laser beam that has been expanded into a band shape is diffusely reflected from the surface of the target. This reflected light is detected by the CMOS sensor. By detecting changes in the position and shape of the reflected band of light, it is possible to measure the position of various points along the surface of the target.

The measurement range and the repeatability of the used sensor are shown in Table 1. The distance between sensor and the surface of the workpiece can vary from $57 \mathrm{~mm}$ to $103 \mathrm{~mm}$ but it should be considered, that the width of the laser beam changes with the distance [5].

Tab. 1: Measurement range and Repeatability of the $3 D$ laser inspection system

\begin{tabular}{|c|c|c|}
\hline & Z-axis & X-axis \\
\hline $\begin{array}{c}\text { Measurement } \\
\text { range }\end{array}$ & $80 \pm 23 \mathrm{~mm}$ & $32 \mathrm{~mm}$ \\
\hline Repeatability & $0.5 \mu \mathrm{m}$ & $10 \mu \mathrm{m}$ \\
\hline
\end{tabular}

\section{Performed tests with the sensor system}

The idea is to integrate the sensor into a milling machine. The process of milling should be interrupted and the optical measuring system should scan the workpiece inside the milling machine. The point of time for starting the measuring process should be selected when it makes sense for the manufacturing process. The dimension of the workpiece should be measured at critical processing points. With information about the dimension of the workpiece the control system of the milling machine should be influenced. By transferring the information about the errors to the milling machine before the next manufacturing step has been started, the milling machine is able to react via software. Depending on the measurement point and concept of integration the adjustment can be made directly or for the next part. Thereby errors during the manufacturing process can be avoided and the quality of the products can be improved.

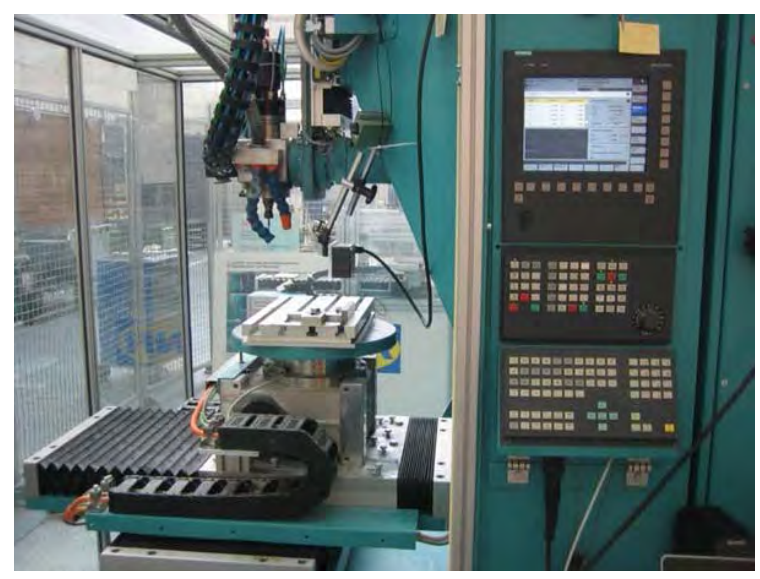

Fig. 1. 5-axis CNC machining center with the integrated laser inspection system.

There are tactile systems on the market, which are able to measure the dimensions of a workpiece inside a milling machine. The reason to use an optical system is the fact, that it is very fast in comparison to a tactile system.

With the experiments, which have been done, should be found out, how exactly the sensor works inside the production area while the machine is running.

It should be performed measurements in 3 dimensions. One series of experiments should be done for the height of the workpiece, one for the width and one for the length. The sensor operates only in two dimensions. Because of that, a further relative movement is necessary. For the test, the movement was realized by the forward movement of the machine table of a milling machine. As test environment a 5-axis CNC machining center was used. Figure 1 shows the milling machine with the integrated sensor.

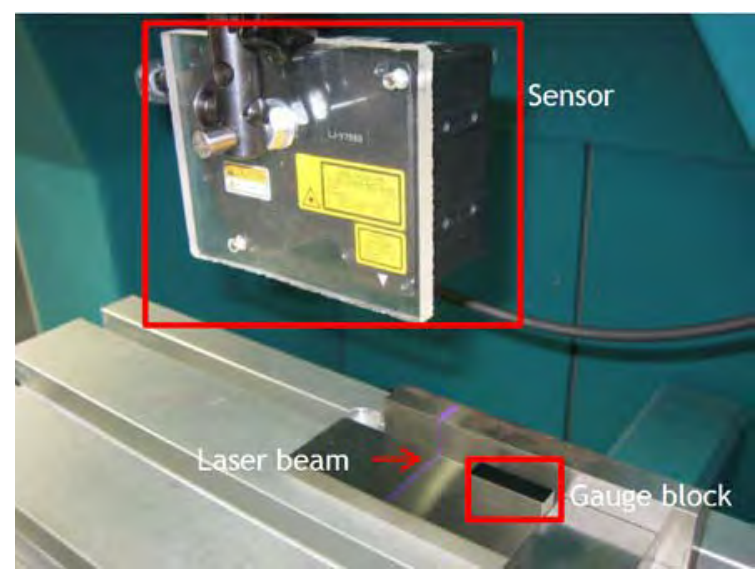

Fig. 2. The experimental setup with the gauge block on the machine table. 
As reference for the measurement, gauge blocks with the dimensions of $4 \mathrm{~mm}, 8 \mathrm{~mm} 10$ $\mathrm{mm}, 14 \mathrm{~mm}, 18 \mathrm{~mm}$ and $23 \mathrm{~mm}$ were used. The gauge blocks were used in each case of the dimension measurement. Figure 2 shows the experimental setup with the gauge block on the machine table in detail.
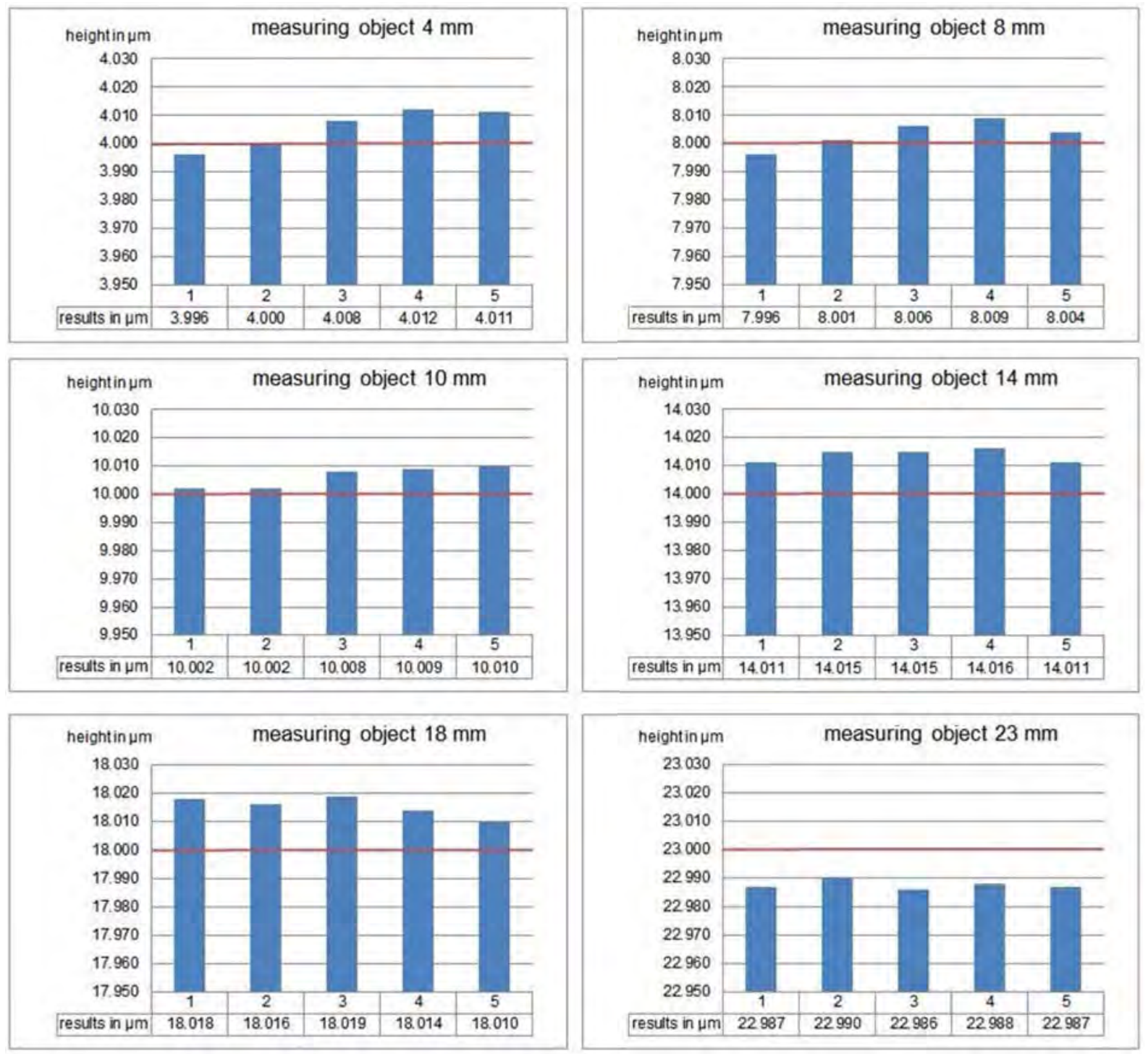

Fig. 3 Verification of height measuring accuracy of the laser scanning system. There are six diagrams, one for each measurement object. Each diagram consists of five blue columns. Each column represents one measurement. The vertical axis is in each case scaled in a range of $+30 \mu m$ to $-50 \mu m$ around the real value of the measured object. The real height of the measuring object is represented by a horizontal red line. Below each respective column the corresponding measurement value is shown.

\section{Results of the tests}

The measurement results for the height and for the width are directly available in the profile data of one measurement. To get results for the length, many successive profile data are needed. With the knowledge about the used sample rate of the sensor and the used forward movement of the machine table, the length of the workpiece can be calculated with the following formula:

$$
\text { length }=\frac{n \times f}{r}
$$

$\mathrm{n}=$ number of equal profiles samples

$\mathrm{f}=$ feed rate $[\mathrm{mm} / \mathrm{s}]$

$r=$ sample rate $[1 / \mathrm{s}]$

In principle, a very high measurement speed can be achieved. It is possible to transmit 64,000 profiles per second. Because of the fact, that the forward movement of the machine table is needed for the measuring process, the speed of the measurement is limited with this movement. 
A result of the previously described experiments was that the measurement error is in the range of $\pm 20 \mu \mathrm{m}$. For example, the results of the height measurement are shown in Figure 3.

The results for the measurement of the length are in the same range as the results of the height measurements. But they depend on the forward movement of the machine table and on the sample rate of the sensor.

The results of the width measurement are much less accurate. The reason for this is the resolution of the sensor, which is $50 \mu \mathrm{m}$ in the width direction (x-direction). Because of that the results cannot be inside a range of a digit micrometer. To receive better results, it is possible to use a second sensor, rotated by $90^{\circ}$ to the first one in a plane orthogonal to the height direction. After that, the data of both sensors should be merged.

\section{Conclusion and future action}

The first studies, show that it is possible to use an optical sensor system in the production area inside a milling machine for the measurement of part geometry. The target of this first studies was to get information about the accuracy of the sensor and about the possibility to get data of all three dimensions from a workpiece. In these studies the measured reference piece had only small dimensions. With a relative movement of the workpiece or a relative movement of the sensor itself, it is possible to enlarge the measurement range. With this possibility the sensor can be used in many different applications.

At the moment these are the first steps of the testing phase. The system should be further improved. If there is a robust measurement system, the next step, to integrate the prepared data of the measurement system into the controller of the milling machine, can be started.

\section{References}

[1] M. Glück, Die "Produktion 2020", Computer \& Automation (2012), Nr. 6, S. 47-50.

[2] R. Schmitt, M. Harding, J. Lose, C. Mersmann, P. Jatzkowski, Anforderungen der Produktion, Verein Deutscher Ingenieure e.V. (Hrsg.), Fertigungsmesstechnik 2020 : TechnologieRoadmap für die Messtechnik in der industriellen Produktion, S.19. Düsseldorf : VDI/VDE, (2011).

[3] W. Osten, E. Garbusi, D. Fleischle, W. Lyda, C. Pruss, R. Reichle, C. Falldorf, "Optical metrology from the laboratory to the real world", Proc. of SPIE 7387 (2010).

[4] W. Lyda, D. Fleischle, M. Gronie, F. Mauch, K. Körner, W. Osten, Herausforderungen und Lösungsansätze für die fertigungsnahe Qualitätskontrolle mittels optischer 3DMesstechnik, 16. GMA/ITG-Fachtagung Sensoren und Messsysteme (2012).

[5] Keyence Corporation of America, High-speed Inline Profile Measurement Device : LJ-V7000 Series, Elmwood Park, (2012). 